\title{
3 Die Strategiewanderung: Veränderungen nachhaltig initiieren und umsetzen
}

Christine Kuch

\subsection{Ergebnisse einer Studie zur Initiierung und Umsetzung von Veränderungsvorhaben}

Veränderungsvorhaben sind nicht immer leicht umzusetzen, insbesondere, wenn es sich um größere Vorhaben handelt. Viele verlaufen im Sand - und das sind leider nicht immer die schlechtesten.

Aber wie kann man es denn anstellen, dass auch über größere Veränderungsvorhaben nicht nur geredet wird, sondern diese tatsächlich angegangen werden?

Diese Frage stand im Zentrum einer Studie, in deren Rahmen in einem dreistufigen, qualitativen Verfahren Strategien zur Initiierung und Umsetzung von Veränderungsvorhaben entwickelt wurden (Kuch 2006). Zunächst wurden Ärzte und Psychotherapeuten zu Problemen bei der Umsetzung von Innovationen in ihrer Organisation interviewt. Zu diesen Problemen formulierten anschließend Organisationsentwickler mit systemtheoretischen und mit lösungsorientiertem Hintergrund hilfreiche Veränderungsstrategien. Die daraus resultierenden, in Cruppen zusammengefassten Veränderungsstrategien wurden schließlich Vertretern von Berufsverbänden, Krankenversicherungen und anderen Institutionen im Gesundheitswesen vorgelegt und auf ihre Vollständigkeit und Angemessenheit hin geprüft und ergänzt.

Die aus diesem Verfahren resultierenden fünfzehn Veränderungsstrategien sind der nachstehenden Abbildung $10 \mathrm{zu}$ entnehmen.

Die einzelnen Strategien werden nachstehend kurz erläutert: 


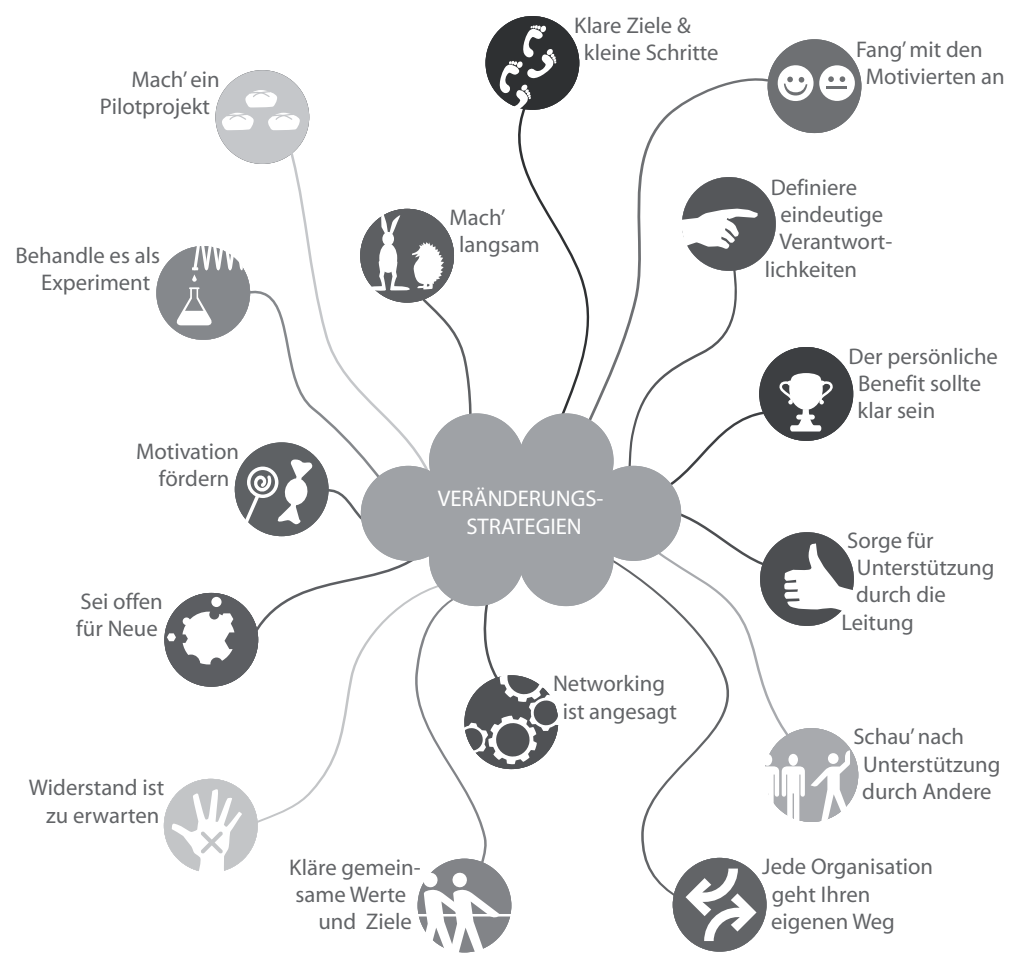

Abb. 10 Veränderungsstrategien, um Innovationen in Gesundheitsorganisationen umzusetzen, (c) Kuch 2007

Klare Ziele und kleine Schritte. Klare Ziele und mögliche Erfolge werden definiert, die erforderlichen und vorhandenen Ressourcen geklärt. Kleine Schritte werden gemacht, die Bemühungen evaluiert und Erfolgsgeschichten verbreitet. Dabei werden auch winzigste Schritte der Veränderung nicht vergessen.

Praktisch heißt das:

- Nicht nur allgemein „Mehr Freundlichkeit im Umgang mit Patienten“ sondern auch schon „Heute morgen das freundliche Lächeln“ wertschätzen.

- Nicht allgemein „Bessere Zusammenarbeit mit der Klinik“ sondern ersten kleinen Schritt „Rückruf bei Anfragen innerhalb von vier Stunden sicherstellen. “definieren (oder noch „kleineren“ Schritt: „Entlassbriefe nicht handschriftlich, sondern immer gedruckt und leserlich.“)

Fang mit den Motivierten an. Veränderung wird grundsätzlich nie übergestülpt. Der Fokus liegt bei den motivierten Personen, deren Beteiligung wird wertgeschätzt. Um in einer größeren Organisation Veränderungen zu implementieren, reicht es, mit motivierten Schlüsselpersonen zu beginnen. In kleineren Organisationen reicht es häufig schon, wenn ein oder zwei Beteiligte mit der Veränderung einfach anfangen und dann sehen, was weiter passiert. 
Praktisch heißt das:

- Wer von den Mitarbeitern Interesse hat, nimmt an gemeinsamer Sitzung teil, und wird dafür gelobt - es wird niemand dazu gezwungen und für eine Nichtteilnahme gerügt.

- Wenn man z. B. die Idee hat, bei Telefonnotizen immer Name, Rückrufnummer und bester Anrufzeitraum zu notieren: selbst anfangen, das konsequent umzusetzen.

Definiere klare Verantwortlichkeiten. Verantwortlichkeiten werden von Anfang an definiert, auch, weil dann die Kommunikation besser klappt: man weiß, wer für was zuständig ist.

Praktisch heißt das:

- Frau Müller kümmert sich hauptverantwortlich um DMP Diabetes - sie koordiniert alle Aktivitäten dazu und hat diesbezüglich auch die volle Weisungsbefugnis.

Der persönliche Nutzen sollte klar sein. Da Menschen letztendlich durch ihre Motive gesteuert werden, sollte der persönliche Nutzen einer Änderung für die Betroffenen klar sein. Um den Nutzen definieren zu können, kann man die Betroffenen danach fragen, was ihre Ziele und Interessen sind, und in wieweit die gewünschten Veränderungen in irgendeiner Art und Weise zur Erfüllung dieser Ziele und Interessen beitragen können.

Praktisch heißt das:

- Noch nicht im Netz beteiligte Ärzte danach fragen, wie sie in 5 Jahren arbeiten möchten und auch danach, ob das Netz ihnen dabei helfen könnte, dies leichter, eher, einfacher, usw. zu erreichen.

Unterstützungen durch die Führung. Falls die Veränderung nicht die Idee des Chefs ist, dann wird die Veränderung mit einer Strategie, wie sie einfach umgesetzt werden kann, präsentiert. Nach einem abgestimmten Zeitraum werden dann die bis dahin erreichten Erfolge präsentiert, da praktischer Erfolg immer noch am meisten überzeugt. Daran denken: Vorgesetzte müssen von der Veränderung nicht begeistert sein, aber sie müssen sie grundsätzlich unterstützen. Praktisch heißt das:

- Nicht erwarten, dass alle Praxisinhaber ob der Idee einer Teambesprechung jubeln, ein kurzes O. k. reicht, um erst einmal drei Teambesprechungen durchzuführen und dann zu schauen, ob sie etwas gebracht haben.

Schau nach Unterstützung durch Andere. Die Bedürfnisse von Patienten, Angehörigen, Zuweisern, usw. werden erhoben. Dann wird geprüft, ob ihnen die gewünschte Veränderung helfen könnte. Zudem kann man immer um (zusätzliche) Finanzierung durch Verbände, Institutionen u.a. anfragen. Schau' auch nach ungewöhnlichen Kooperationspartnern: Was kann der Zoo, das Museum, die Medizintechnik zum eigenen Vorhaben beitragen? 
Praktisch heißt das:

Die Zusammenarbeit mit dem Kindergarten und wöchentliche gemeinsame Workshops von Kindern und Heimbewohnern kann ebenfalls zu mehr Aktivität einiger Heimbewohner führen.

Jede Organisation braucht ihren eigenen Weg. Da jede Einrichtung unterschiedlich ist, muss ein individuelles Vorgehen geplant werden. Hilfreiche Fragen dabei sind: Wie wurde in der Vergangenheit bei uns schon erfolgreich eine umfassende Veränderung umgesetzt? Was hat dabei funktioniert? Zudem ist hilfreich, den Prozess, in dem die Veränderung erwünscht ist, zu definieren und dann den Prozess insgesamt vorsichtig zu verändern.

Praktisch heißt das:

- Erst mal die Frau vom Chef für die Veränderung gewinnen, dann läuft's bei uns.

- Nicht isoliert die Wartezeit im Wartezimmer verändern, sondern den Prozess „Patienten betritt die Praxis“ bis zu „Patienten spricht mit Arzt im Behandlungszimmer" dahingehend optimieren, dass das Gespräch im Arztzimmer möglichst optimal verlaufen kann.

Networking als Daueraufgabe. Networking vergrößert die Chance erfolgreicher Umsetzung. Fördere Netzwerke, die mit dem zu verändernden Prozess in Verbindung stehen, fördere auch die Vernetzung der Beteiligten untereinander.

Praktisch heißt das:

- Im Team darüber reden, wer welche Gemeinsamkeiten mit wem hat und kurze Pausen und Pläusche der Mitarbeiter untereinander fördern.

- Kaffeemaschine auch im Keller bei der Physiotherapie aufstellen - seitdem haben die Mitarbeiter einen besseren Austausch untereinander.

Kläre gemeinsame Werte und Ziele. Veränderungen werden nur umgesetzt, wenn sie mit den vorherrschenden Werten und Normen vereinbar sind. Da die Werte von Gesundheitsorganisationen meist noch immer nicht explizit definiert sind, sollten sie geklärt werden. Da Werte nicht verordnet werden können, werden sie gemeinsam im gesamten Team definiert. Sie können dabei top down von oben nach unten entwickelt werden oder man entwickelt die Werte in den einzelnen Berufsgruppen und fügt sie dann in einer gemeinsamen Besprechung zusammen.

Praktisch heißt das:

- Ein Leitbild zu „Wie wollen wir in fünf Jahren arbeiten? Für wen? Wofür stehen wir? Was bieten wir an und was nicht?" wird im gesamten Team erarbeitet, indem alle Mitarbeiter auf Karteikarten schreiben, was Ihnen dazu wichtig erscheint. Diese Karten werden im Anschluss daran in Gruppen zusammengefasst und diskutiert. Auf Grundlage dieser Vorarbeit wird schließlich ein schriftliches Leitbild verfasst, das z. B. auch Patienten gegenüber veröffentlicht werden kann. 
Widerstand ist zu erwarten. Änderungen werden nicht verordnet, da dies zu teilweise begründetem Widerstand führen kann. Es lohnt sich, die Ursachen des Widerstandes mit den Betreffenden zu klären und sie nach möglichen Vorschlägen für das Veränderungsvorhaben zu fragen, um die Qualität der Veränderung zu erhöhen. Falls man Andere von einer Sache überzeugen möchte, sollte man daran denken, dass Erfolg und Nutzen Menschen überzeugt, nicht Druck.

Praktisch heißt das:

- Herr Dr. Meier fand die Idee, so viel Aufwand um QM zu machen, eher bescheiden. Schließlich hatte er in der Klinik schon mitbekommen, dass das vor allem ein großer Papiertiger ist. Herr Dr. Meier war begeistert von den ersten Veränderungen, weil er nicht erwartet hatte, dass die Mitarbeiter so engagiert mitmachen und so tolle Ideen haben würden, und dass die Terminvergabe unter den fünf Kollegen tatsächlich geklärt werden könnte. Außerdem war ihm vorher nicht bewusst, dass es Mitarbeiter gibt, die den Eindruck haben, bisher nicht wirklich gehört worden zu sein(und sich ernsthaft über diese Veränderung freuten).

Sei offen für neue Mitglieder. Das eigene Vorhaben wird nie wie ein „Closed Shop“ behandelt, sondern ist immer offen für neue Mitwirkende, auch wenn diese zunächst ungewöhnlich erscheinen mögen. Zudem sind Änderungen Eigentum der Betroffenen. Beispielsweise bestehen Wahlmöglichkeiten bei der Umsetzung der Veränderungen. In jede Richtung wird offen über die avisierten Änderungen kommuniziert, dabei wird die Sprache der Betroffenen genutzt.

Praktisch heißt das:

- Im Ärztenetz empfiehlt es sich, den Start neuer Projekte immer kommunizieren und um aktive Mithilfe, Anregungen und Kritik zu bitten. Dies gilt selbstverständlich auch für erreichte Teilergebnisse. Die Mitglieder müssen (potenziell) die Möglichkeit haben, sich zu beteiligen. Bei der Umsetzung des Projektergebnisses, beispielsweise der Implementierung eines neuen Behandlungspfades, sollten z. B. Wahlmöglichkeiten in Bezug auf die Methode, wie der Behandlungspfad in die einzelne Praxis implementiert wird, angeboten werden.

- Für die Praxis bedeutet diese Strategie beispielsweise, dass es nicht nur einen Weg gibt, das Klima im Team zu verbessern. Jeder sollte über mögliche Ansatzpunkte zur Verbesserung mitdenken und sie einbringen können. Außerdem sollten Wahlmöglichkeiten bestehen: nicht jedes Teammitglied freut sich über einen regelmäßigen Kegelabend, es sollten mehrere Angebote überlegt werden, den Teamgeist zu stärken.

Unterstütze Motivation. Um die Motivation zu unterstützen, insbesondere nach einem Veränderungsstart, werden schon kleinste Fortschritte und Erfolge wertgeschätzt. Es lohnt sich auch, die Lösungen der Betroffenen zu imple- 
mentieren: Was hat in dem betreffenden Prozess schon gut geklappt? Was kann leicht im Sinne der gewünschten Verbesserung verändert werden?

Praktisch heißt das:

- Dr. Schmidt will anfangen, Patienten stärker in die Therapieplanung einzubeziehen. Er staffelt seine Ziele: Zunächst wird er sich freuen, wenn ihm das bei dem ersten Patienten gelingt. Das nächste Ziel besteht darin, noch mehr Patienten mit Diabetes in die Entscheidung einzubeziehen. Gelingt ihm auch dies, will er bei weiteren Patienten mit anderen, auch verhaltensabhängigen Erkrankungen sein Vorgehen erproben.

- Die Arbeitsleistung des Teams am Tresen ist sehr unterschiedlich. Aber die Einnahme und Dokumentation der Praxisgebühr klappt bei allen. Wieso funktioniert das?

Behandle es als Experiment. Die Umsetzung von Veränderungen wird als Experiment behandelt. Es ist leichter, viele kleine Experimente zu unternehmen und anhand derer Neues zu lernen: „Klein und schön“ anstelle von „Perfekt und nie“. Praktisch heißt das:

- Patienten beschweren sich über entstehende Wartezeiten. Man hat aber gegenwärtig weder die Möglichkeit, die Wartezeiten wesentlich zu reduzieren, noch ausreichende finanzielle Mittel, den Wartebereich durch innenarchitektonische Raffinessen, oder interaktive Medien „aufzupeppen“. Statt darauf zu warten, dass irgendwann einmal etwas passieren wird, kann man Patienten nach Verbesserungsideen und -wünschen befragen (einige Patienten kommen in vielen Wartezimmern herum und haben eine Menge Ideen). Im Folgenden kann sich jeder Mitarbeiter eine Maßnahme überlegen, die er alleine und aufwandsarm umsetzen kann, damit die Situation für die Patienten (und vielleicht auch für den Mitarbeiter) leichter wird.

Mach ein Pilotprojekt. Ein Pilotprojekt sollte von Mitarbeitern, die schon von sich aus am Thema interessiert sind, an einem solchen Projekt zu arbeiten, durchgeführt werden. Dabei wird darauf geachtet, was bereits gut funktioniert und es werden nur kleine Veränderungen in die gewünschte Richtung formuliert. Der Pilotcharakter wird im Unternehmen kommuniziert, damit die Beteiligten wissen, dass ungewöhnliche Dinge ausprobiert werden können. Es wird viel interne PR gemacht, damit jeder weiß, dass das Pilotprojekt durchgeführt wird. Für Input und Kritik von anderen ist das Pilotprojekt immer offen. Abschließend erfolgt eine Evaluation und die Resultate werden allen übermittelt.

Praktisch heißt das:

- Man muss nicht alles auf einmal umorganisieren. Man könnte zum Beispiel erst einmal damit anfangen, die Abläufe im Labor unter die Lupe zu nehmen und erste Erfolge, wie $z$. B. eine schnellere Bearbeitung, eine fehlerfreiere Dokumentation, ein noch besserer Schutz der Patienten- 
daten vor unbefugtem Zugriff, zu feiern. Dabei kann man dann überlegen: Was davon können wir auf andere Bereiche der Praxis übertragen? Wo könnte etwas Ähnliches funktionieren?

- In Ärztenetzen muss nicht jede Praxis alles machen, gerade hier kann man viel voneinander profitieren. Erfolge einzelner Praxen werden im Netz kommuniziert, damit auch andere merken, dass sich die Mühe, z. B. bei der Bearbeitung eines Prozesses, oder bei der Verbesserung der Zusammenarbeit im Team, tatsächlich lohnt.

Mach langsam. Menschen, die Innovationen umsetzen wollen, sollten daran denken, dass die Dinge nur organisch wachsen und dass Veränderung Zeit braucht. Man sollte nicht zu viel auf einmal erwarten

Praktisch heißt das:

- Nicht frustriert sein, wenn nicht alle beim sektorenübergreifenden Behandlungsplan konsequent mitmachen. Stattdessen sorgsam evaluieren, an welchen Stellen sich an die Vorgaben gehalten wird und an welchen Stellen nicht. Da, wo es nicht bei allen klappt, schauen, wie diejenigen, die sich an die Vorgaben halten, dies erreichen.

- Eine Ärztin fragte andere Kollegen an, ob sie Interesse an einem kollegialen Austausch hätten. Drei Kollegen waren interessiert und zwei davon nahmen dann an regelmäßigen Treffen teil. Drei Jahre später hatte eine Gruppe von 15 Ärzten, zu denen auch Klinikärzte gehörten, begonnen, einen sektorenübergreifenden Behandlungsplan zu erstellen.

\section{Aufgabe 12 für anwendungsfreudige Leser}

Überlegen Sie ein Vorhaben, das Sie derzeit beschäftigt und schreiben Sie es bitte auf.

Lesen Sie nun alle Strategien noch einmal durch. Zu den Strategien, die Sie aus irgendwelchen Gründen ansprechen, notieren Sie bitte, was das für Ihr Vorhaben bedeutet. Was können (und sollten) Sie als Nächstes tun?

\subsection{Das Instrument: „Strategiewanderung“}

Um diese Veränderungsstrategien praktisch nutzbar zu machen, wurde ein Instrument entwickelt, das die Strategien mit der Formulierung von konkreten Handlungsschritten verbindet, die „Strategiewanderung“. Diese ist ein Instrument zur Unterstützung von Veränderungsprozessen. Sie hilft dem gesamten Praxisteam, passende Strategien für ein Veränderungsvorhaben strukturiert und pragmatisch zu definieren, ist aber auch für praxisübergreifende Veränderungsprozesse nutzbar.

Zu Beginn der Strategiewanderung wird zunächst das angestrebte Ziel konkretisiert. Anschließend verteilen sich die Beteiligten im Kreis um auf dem Boden ausgelegte Blätter, auf denen jeweils eine Strategie mit dem passenden Bild und kurzen erklärenden Anmerkungen abgebildet sind. 
Nun fängt die „Wanderung“ an: Eine Minute lang schauen sich die Teilnehmenden die Strategien an und überlegen, welche der Strategien sie anspricht, um sich dem angestrebten Ziel zu nähern. Nach einer Minute begeben sich die Teilnehmenden zu der Strategie, mit der sie bislang am meisten anfangen konnten.

Wie bei jeder Wanderung macht man auch mal Pause und schreibt eine Postkarte: In diesem Schritt haben die Teilnehmenden zweieinhalb Minuten Zeit, um auf ausliegenden Karten die konkreten Schritte zu formulieren, die ihnen in Bezug auf das angestrebte Ziel eingefallen sind.

Im nachfolgenden Schritt, dem Plausch, haben die Teilnehmenden drei Minuten Zeit, um sich mit ihrem Nachbarn über die gewählten Strategien und die definierten konkreten Maßnahmen auszutauschen (und sich dafür kräftig zu loben).

Die Wanderung wird noch einmal wiederholt, die Teilnehmenden erwandern für sie passende Strategien, formulieren konkrete Handlungsschritte und tauschen sich darüber mit ihren Nachbarn aus.

Abschließend werden die ausgewählten Strategien zusammen mit den konkreten Maßnahmen noch einmal angeschaut und es wird überlegt, ob und in welcher Reihenfolge die formulierten Maßnahmen von wem durchgeführt werden und wann eine Evaluation erfolgt.

Der gesamte Zeitaufwand beträgt zwischen einer halben Stunde und eineinhalb Stunden. Das Instrument wurde bislang erfolgreich in Deutschland, Großbritannien, Schweden, Österreich und in der Schweiz eingesetzt. Eine der häufigsten Rückmeldungen von Teilnehmenden ist, dass man, ohne in zeitaufwändiges Gerede abzugleiten, sehr schnell konkrete Handlungsschritte gemeinsam formulieren und jeder sich dabei einbringen kann.

Eine detaillierte Beschreibung des Vorgehens ist bei Kuch (2008) zu finden, bei Interesse kann das passende Material bei der Autorin bestellt werden.

\section{Literatur}

Kuch C: Initiating Organisational Development. In: Lueger, Korn: Solution-focused management. München: Rainer Hampp Verlag 2006: 249-257

Kuch C: Strategie Wanderung. In: Röhrig: Solution tools. Bonn: managerSeminare Verlag 2008: 194-199 